\title{
The Oxygen Problem
}

\author{
Michael S. Bessell \\ Mount Stromlo Observatory, Cotter Rd, ACT 2611, Australia
}

\begin{abstract}
Permitted OI, forbidden [OI], electronic $A \Sigma-X \Pi$ UV OH and vib-rot IR OH lines often infer different oxygen abundances for cool stars. Which species should one use to determine the oxygen abundance in cool stars and how does one reconcile the different results for different species? Although there are uncertainties and systematic differences in the molecular $g f$ values used by different authors, the main reason for the different results lies in the 1-D model atmosphere analyses that are used.
\end{abstract}

\section{Introduction}

Israelian et al. (1998) and Boesgaard et al. (1999) using the permitted OI and the UV OH lines in mostly halo subdwarfs, have derived $[\mathrm{O} / \mathrm{Fe}]$ versus $[\mathrm{Fe} / \mathrm{H}]$ ratios that show oxygen overabundances relative to iron continue to increase as $\mathrm{Fe} / \mathrm{H}$ is decreased. If this result is true, the behaviour of oxygen is different from that of other alpha elements, such as $\mathrm{Ca}$ and $\mathrm{Ti}$, whose overabundances relative to iron, $[\alpha / \mathrm{Fe}]$, reach a plateau of about +0.5 dex for $[\mathrm{Fe} / \mathrm{H}] \approx-1.0$ and leads to important consequences for the nuclear synthesis in massive stars. However, there are important differences between the various atomic and molecular species used for these abundance determinations that need examination before the unexpectedly high $\mathrm{O} / \mathrm{Fe}$ ratios can be accepted at face value.

\section{Discussion}

\subsection{Atomic and molecular species differences}

Unlike other alpha elements, the various oxygen species used in the investigations are formed at different levels in the atmosphere and with a variety of strengths. This makes the lines very sensitive to temperature and gravity. The high excitation of the permitted OI lines means that the lines are formed in the hotter levels in the atmospheres of the hotter stars. On the other hand, the molecular $\mathrm{OH}$ lines are formed in the cooler layers of the cooler stars. Finally, the low excitation forbidden [OI] lines have extremely low probability and so are mainly seen in the cooler and deeper atmospheres of giants. In contradistinction, other $\alpha$ elements, such as $\mathrm{Ca}$ and $\mathrm{Ti}$, together with $\mathrm{Fe}$ have similar levels of ionization and excitation so their lines are formed in similar regions of the atmosphere and the derived abundance ratios are thus relatively insensitive to temperature.

It is noteworthy that the oxygen abundances in halo giants derived from the [OI] line over the past 25 years have not shown the large overabundances derived 
recently for halo dwarfs. This implies either that oxygen is processed in giant stars, or more likely, that there is some systematic difference from the analyses of halo giants and dwarfs. Recent work on [OI] in giant stars by Fulbright \& Kraft (1999) and now on IR OH lines in giants by Melendez \& Barbuy (this meeting) supports the normal behaviour of oxygen in halo giants.

Not all $\mathrm{O}$ species can be seen in all halo stars. Permitted OI is only observable in the hottest dwarfs and then is only reliable for $[\mathrm{Fe} / \mathrm{H}] \geq-2$. Forbidden [OI] can usually be measured in halo giants with $[\mathrm{Fe} / \mathrm{H}] \geq-2$ but is only seen in cool dwarfs with $[\mathrm{Fe} / \mathrm{H}] \geq-1$. UV OH is the only $\mathrm{O}$ species that can be seen in the most metal deficient (cool) stars with $[\mathrm{Fe} / \mathrm{H}]=-4$ so it is imperative to ensure that $\mathrm{OH}$ abundances are understood.

\subsection{Systematic differences between giants and dwarfs}

One obvious difference between halo giants and halo dwarfs are their likely temperatures. Consideration of an isochrone of about 12 billion years shows that in a given apparent magnitude range most stars will be main sequence dwarfs, turnoff stars and subgiants with temperatures between $5000 \mathrm{~K}$ and $6000 \mathrm{~K}$ and red-giants with temperatures between $5200 \mathrm{~K}$ and $4200 \mathrm{~K}$. A typical halo giant that is observed will therefore be about $1000 \mathrm{~K}$ cooler than a typical halo dwarf. Such large temperature differences suggest the possibility of systematic differences in results for giants and dwarfs.

\subsection{Model atmosphere problems}

Most of the problems in the analysis of subdwarfs and subgiants almost certainly arise from use of $1 D$ model atmospheres. Real stellar atmospheres for cool stars are inhomogeneous and there is no unique temperature versus depth (tau) relation. This can be seen in the Sun (e.g. Åke Nordlund and collaborators). Work is being done by this group on subdwarfs and subgiants; see Asplund (2001).

The empirical Holweger-Muller 1D model structure has done a reasonable job for many elements and excitations, but Grevesse \& Sauval (1999) have shown that cooler temperatures at small depths better fit low excitation FeI lines. However, such a model structure does not fit the solar IR OH lines. These are examples of the non-uniqueness of $1 \mathrm{D}$ temperature-depth relations in solar-type stars. Metal-deficient stars will show even greater deviations.

Careful differential analyses have normally permitted good abundance results to be obtained for cool stars using 1D model atmospheres, but this was when species of similar excitation and ionization were used. The use of high excitation OI lines together with molecular $\mathrm{OH}$ lines pushes such differential analyses to the limits and greatly restricts the stellar temperature range one can work across and also the species used for comparison.

Problems evident in using cool dwarf 1D model atmospheres are

- different model $\ell / H$ needed to fit observed hydrogen lines and continuum.

- convective overshoot or no-overshoot assumption in models

- color-temperature relations differ with overshoot and mixing length values

- ionization equilibria difficult to achieve for true effective gravity

- non-LTE effects for minority ionization species (overionization)

- OI, [OI], $\mathrm{OH}$ often give different results 
It appears that the 1D no-overshoot models of Castelli/Kurucz (Bessell et al. 1998; Castelli, 1999) give the most consistent results for colors and hydrogen lines but more testing is needed in the line analysis for metal-poor stars.

\subsection{OH gf values and line lists}

There are limitations associated with using the UV OH lines, mainly suspected uncertainties of at least 0.3 dex in the absolute $g f$ values and larger uncertainties for lines from higher $J$ levels and different vibrational levels. Some authors have used the theoretical values unadjusted while others have used solar $\mathrm{OH}$ lines to normalize $g f$ values for the different vibrational bands. Use of the few reasonably clean solar lines is still problematic as the lines are mostly strong and blended and because of the uncertain solar UV opacity. For accurate absolute $g f$ values one must know the electronic moment of the $\mathrm{OH}$ molecule for a large range of internuclear distances. This is difficult to determine experimentally but we look forward to theoretical electronic transition moments being calculated by Nickolay Dobrodney (Heidelberg) who has already successfully computed accurate $\mathrm{TiO}$ and VO electronic transitions. There are currently at least four independent sources for UV OH line lists, wavelengths, energy levels, from at least four independent sources: Goldman \& Gillis (1981), Gillis et al. (2000), Goldman (2000), Luque \& Crosley (1998): http://www.sri.com/CEM/LIFBASE, Kurucz (1993) and Nissen et al. (1994). Comparison of these lists shows that there is good agreement between Goldman and LIFBASE for low $J$ values, and for lines between 3120 and $3150 \AA$, a good region in which to work.

\section{Conclusions}

Because 1D model atmospheres are not good approximations for the real atmospheres of cool stars it is likely that systematic differences in abundances occur with atomic and molecular species that are very sensitive to the temperature.

\section{References}

Asplund, M. 2001, these proceedings

Bessell, M.S. et al. 1998, A\&A, 333, 231

Boesgaard, A.M. et al. 1999, AJ, 117, 1549

Castelli, F. 1999, A\&A, 346, 564

Fulbright J.P. \& Kraft, R.P. 1999, AJ, 118, 527

Gillis, J.R. et al. 2000, JQSRT, in press

Goldman, A. \& Gillis, J.R. 1981, JQSRT, 25, 111

Goldman A. 2000 private communication

Grevesse, N. \& Sauval, A.J. 1999, A\&A, 347, 348

Israelian, G. et al. 1998, ApJ, 507, 8051

Kurucz, R.L. 1993, CDROM 15

Luque, J. \& Crosley, D.R. 1998, JChPh, 109, 439

Nissen P. et al. 1994, A\&A, 285, 440 